\title{
CRANIOPLASTIA DE FRONTAL COM MALHA DE TITÂNIO APÓS CRANIECTOMIA DESCOMPRESSIVA
}

\author{
FRONTAL CRANIOPLASTY WITH TITANIUM MESH AFTER DECOMPRESSIVE CRANIECTOMY
}

\author{
Laís Inês Silva Cardoso ${ }^{1}$, Sérgio Éberson da Silva Maia ${ }^{2}$, João Marques Mendes Neto ${ }^{3}$, Renato da Costa Ribeiro ${ }^{4}$, \\ Carlos Eduardo Mendonça Batista ${ }^{5}$, Simei André da Silva Rodrigues Freire ${ }^{6}$.
}

1 Residente em Cirurgia e Traumatologia Bucomaxilofacial, Hospital Universitário, Universidade Federal do Piauí (HU-UFPI), Cirurgiã-Dentista Universidade Federal do Maranhão (UFMA), Brasil.

2 Cirurgião Dentista pela Unileão, Juazeiro do Norte - CE. Cirurgião Bucomaxilofacial pela Universidade Federal do Piauí (UFPI), Brasil.

3 Cirurgião Dentista pela UESPI, Parnaíba - PI. Cirurgião Bucomaxilofacial pela Universidade Federal do Piauí (UFPI), Brasil.

4 Cirurgião Bucomaxilofacial pelo Hospital Municipal Dr. Mário Gatti, mestre em Cirurgia e Traumatologia Bucomaxilofacial pela Faculdade de Odontologia de Piracicaba - FOP/UNICAMP, preceptor do Hospital Universitário (UFPI), Brasil.

5 Cirurgião Bucomaxilofacial e mestre em Odontologia pela Universidade Federal do Piauí - UFPI, preceptor do Hospital Universitário (HU-UFPI), Brasil.

6 Cirurgião Bucomaxilofacial pelo CFO, mestre e doutor em Cirurgia e Traumatologia Bucomaxilofacial pela Faculdade de Odontologia de Piracicaba - FOP/UNICAMP, preceptor do Hospital Universitário (HU-UFPI), Brasil.

\section{RESUMO}

O osso frontal compõe a região anterior do crânio e suas principais funções são proteger o encéfalo de traumas diretos e infecções. Na ocorrência de perda de parte desse osso, há uma desarmonia funcional e anatômica, sendo indicada sua reconstrução. O objetivo deste trabalho é apresentar um relato de cranioplastia de osso frontal, decorrente de uma craniectomia descompressiva de urgência, utilizando como material a malha de titânio. Relato do caso: Paciente do gênero masculino, 32 anos, apresentou-se ao serviço de cirurgia bucomaxilofacial do Hospital Universitário da Universidade Federal do Piauí com queixa de "sofri um acidente e afundou meu rosto". Vítima de acidente motociclístico há cerca de 12 meses antes da consulta citada, foi submetido à uma cirurgia do tipo craniectomia descompressiva, onde foi removido parte do seguimento ósseo frontal cominuído durante o trauma, permanecendo com defeito ósseo resultante da abordagem neurocirúrgica, abrangendo as corticais externa e interna do osso frontal. A partir da queixa principal, resultados dos exames clínico e de imagem foi proposto a cranioplastia para recontrução do osso frontal com malha de titânio. Para o embasamento teórico do caso foi realizado levantamento bibliográfico acerca do tema na base PubMed, utilizando os descritores: Fratura do crânio com afundamento; Osso frontal; Titânio, foram selecionados artigos que apresentaram discussão referente à escolha do material de reconstrução. Resultados: a instalação e adaptação da malha de titanio possibilitou a correção do defeito na região frontal, favorecendo a proteção do encefalo e restabelecendo a estética do terco superior da face. Conclusão: Diversos materiais de reconstrução são citados na literatura, entretanto, nenhum contempla todas as caracteristicas ideaias para todos os casos. O titânio é considerado uma excelente opção em termos de força, baixos índices de infecção, alta biocompatibilidade e por ser biologicamente inerte. A cranioplastia para reconstrução do osso frontal possibilita o restabelecimento estético e funcional nos casos de defeitos extensos onde a perda do arcabouço de proteção 
do encefalo além de alteração do contorno do terco superior da face gera tambem uma suceptibilidade a injúrias das estruturas intracranianas.

DESCRITORES: Fratura do crânio com afundamento; Osso frontal; Titânio.

\section{ABSTRACT}

The frontal bone is part of the anterior cranium and its main functions are to protect the brain from direct trauma and infections. In the event of loss of part of this bone, there is a functional and anatomical disharmony, needing its reconstruction. The aim of this paper is to present a report of frontal bone cranioplasty, the defect was the result of an urgent decompressive craniectomy, using a titanium mesh as material. Case report: A 32-year-old male patient presented to the maxillofacial surgery service of the University Hospital of the Federal University of Piaui with the complaint of "I had an accident that depressed my face". He was victim of a motorcycle accident about 12 months before the mentioned appointment, a decompressive craniectomy surgery was performed, and the comminuted segment of the frontal bone fractured during the trauma was removed, remaining a bone defect resulted from the neurosurgical approach, including cortical external and internal frontal bone. Based on the main complaint, clinical and imaging exam results, cranioplasty was proposed to reconstruct the frontal bone with titanium mesh. For the theoretical basis of the case, a bibliographic survey about the theme was carried out in the PubMed database, using the descriptors: Blood fracture with sinking; Frontal bone; Titanium, articles were selected that separated regarding the choice of reconstruction material. Results: the installation and adaptation of the titanium mesh enabled a correction of the defect in the frontal region, favoring the protection of the brain and restoring the aesthetics of the upper third of the face. Conclusion: Several reconstruction materials are mentioned in the literature, however, none contemplates all the characteristic ideas for all cases. Titanium is considered an excellent option in terms of strength, low infection rates, high biocompatibility, and biologically inert. Reconstruction of the frontal bone allows the restoration of aesthetics and functionality in cases of extensive defects where the loss of the brain's protective framework, in addition to changing the contour of the upper third of the face, also generates a susceptibility to injuries such as intracranial structures.

KEYWORDS: Skull Fracture, depressed; Frontal bone; Titanium.

\section{Como citar este artigo (Vancouver):}

Cardoso LIS, Maia SES, Mendes Neto JM, Ribeiro RC, Batista CEM, Freire SASR. Cranioplastia de frontal com malha de titânio após craniectomia descompressiva. J. Ciênc. Saúde [internet]. 2021 [acesso em: dia mês abreviado ano]; JCS HU-UFPI. maio-ago. 2021; 4(2):26-34. Disponível em: DOI: https://doi.org/10.26694/jcshuufpi.v4i2.1003

\section{(cc)BY}

Esta obra está licenciada sob uma Licença Creative Commons Atribuição 4.0 Internacional. 


\section{INTRODUÇÃO}

Os defeitos de osso frontal podem ser resultantes de ressecção de tumores, osteomielites, sequela de cirurgias neurológicas (como craniectomias descompressivas), e traumas, sendo considerada uma fratura relativamente incomum (representa 6 a 12\% de todas as fraturas craniofaciais). Quando resultante de trauma, a causa mais comum são os acidentes com veículos motores, seguida de agressões físicas e quedas. Como a parede anterior do osso frontal é capaz de suportar energia de alta intensidade, geralmente a ocorrência desta fratura está associada a injúrias intracranianas ${ }^{(1,2,3)}$.

A função primária do crânio é abrigar e proteger o cérebro, de maneira que qualquer pequeno defeito ósseo pode tornar o paciente vulnerável a traumas diretos neste órgão, ser uma via para infecções, alterar a dinâmica de produção do líquido cérebroespinhal, aumentar o risco de formação de pseudomeningoceles, além do defeito estético prontamente perceptível ${ }^{(2,4)}$.

A craniectomia descompressiva é um procedimento tradicional e baseado em evidências, indicada para os casos onde há aumento da pressão intra-craniana não responsiva ao tratamento com medicamentos. $\mathrm{O}$ procedimento consiste na remoção de uma janela óssea, de maneira a permitir que o aumento de líquido céberoespinhal ou coleção de sangue, que está aumentando a pressão cerebral, drenem externamente, e o equilíbrio fisiológico seja restabelecido ${ }^{(5,6)}$.

Diversas abordagens cirúrgicas são relatadas na literatura para a reconstrução de defeitos da parede anterior do osso frontal, com aplicação de materiais distintos, tais como: polimetilmetacrilato (PMMA), placas e malhas de titanio, osso autógeno, polietereter-acetona (PEEK), todos paresntam vantagens e desvantagen, demandando um correto planejamento e individualizaçao da abordagem de cada caso, observando o custo-benefício de cada material e os objetivos do tratamento proposto $(7,8)$.

O objetivo deste estudo é apresentar um relato de caso de cranioplastia para reconstrução da parede anterior do osso frontal utilizando como material a malha de titânio para tratamento de sequela de craniectomia descompressiva. reconstrução. Comitê de Ética CAAE: 40341120.9.0000.8050.

\section{RELATO DE CASO}

Paciente do gênero masculino, 32 anos, oriundo do interior do estado do Piauí, apresentou-se ao serviço de cirurgia bucomaxilofacial do Hospital Universitário da Universidade Federal do Piauí com queixa de "sofri um acidente e afundou meu rosto". O histórico do paciente refere-se a um acidente motociclístico há cerca de 12 meses antes da consulta citada, este foi submetido à uma cirurgia do tipo craniectomia descompressiva, na qual foi removido parte do seguimento ósseo frontal cominuído durante o trauma, e com objetivo de reestabelecer a fisiologia intra-craniana. Na Figura 1. A, $B$ e $C$ observa-se por meio de imagens tomográficas o defeito ósseo resultante da craniectomia descompressiva, a qual abrangeu as corticais externa e interna do osso frontal.
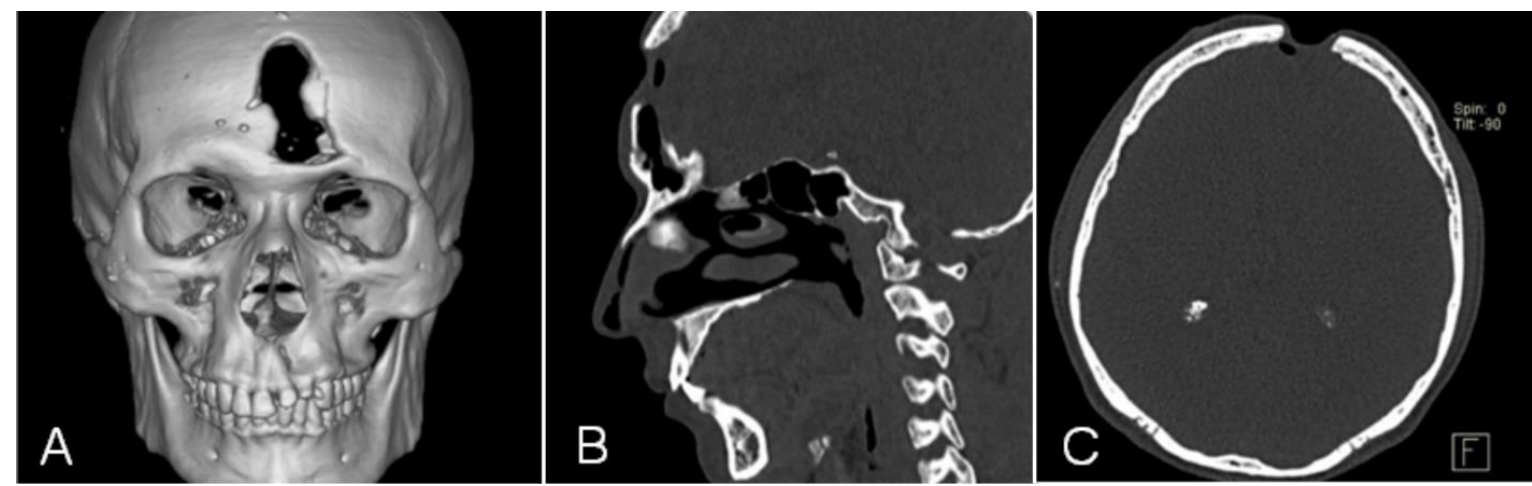

Figura 1 - Imagens pré-operatórias de tomografia computadorizada da face, evidenciando o defeito ósseo em região frontal mediana, com perda óssea das corticais externa e interna. A - reconstrução 3D, B - corte sagital e C - corte axial. 
O paciente nega alterações sistêmicas, vícios, e uso contínuo de medicamentos. Ao exame clínico foi possível observar um afundamento na região frontal, atingindo os limites do seio frontal, evidenciada na
Figura 2. A em vista frontal e B em vista ínfero-superior. $O$ paciente também nega qualquer sintomatologia relacionada ao sistema nervoso, como convulsões, e alteração dos níveis de consciência.

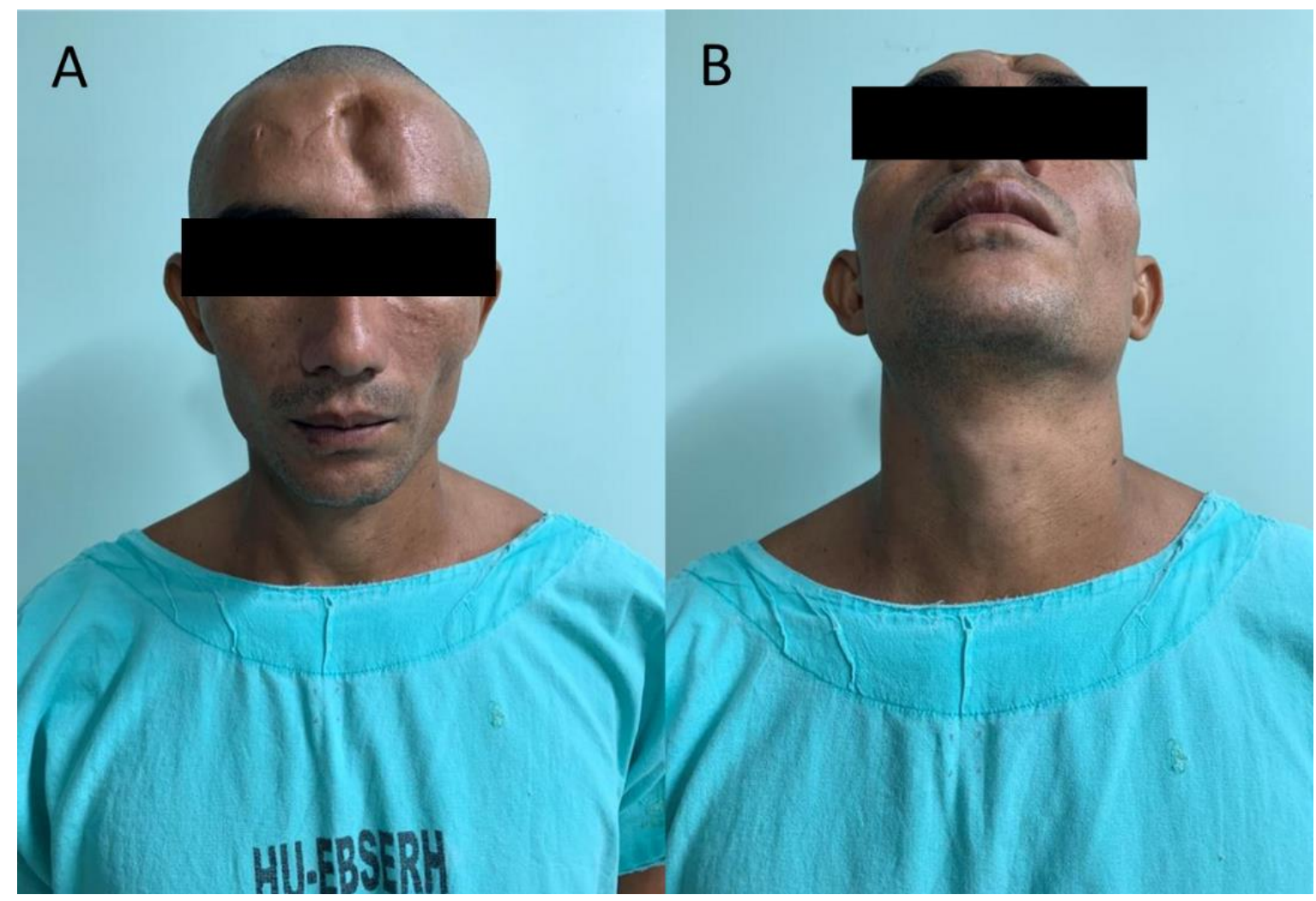

Figura 2 - Paciente em avaliação clínica pré-operatória permite observar defeito côncavo em região frontal média, com sutil deslocamento para a esquerda. A. Vista frontal; B. Vista ínfero- superior.

Foi solicitada uma interconsulta com a equipe da neurocirurgia, de maneira a garantir a segurança do paciente na realização do procedimento de cranioplastia. A especialidade liberou o paciente da necessidade de qualquer medicamento por parte deles, e manteve o acompanhamento do paciente em caráter ambulatorial apenas se necessário.

Desta maneira, como plano de tratamento optouse pela reconstrução de osso frontal com o uso de tela de titânio de estoque, por ser um material de boa manipulação, modelável, e prontamente disponível pelo hospital. O procedimento foi realizado sob anestesia geral.

O acesso de escolha foi a incisão bicoronal, realizada sob as recomendações clássicas do professor Edward Ellis III e Michael F. Zide ${ }^{(7)}$, de maneira a proteger a fáscia temporal e seu conteúdo, manter o pericrânio íntegro e a ser incisado apenas na região próxima ao defeito ósseo, e ao rebater o retalho, cuidadosamente descolar o tecido circundante a região de craniectomia, de maneira a não expor a dura máter. A sequência cirúrgica é apresentada na Figura 3. A. Demarcação da incisão bicoronal, levando em 
consideração sua posição ântero-posterior respeitando o "pico de viúva" presente em pacientes que tem calvície, além da presença de demarcações verticais para o reposicionamento em posição adequada do retalho; B. Retalho coronal evertido, periósteo mantido em posição, sendo incisado apenas na área de interesse cirúrgico; C. Periósteo em evidência, respeitando o contorno do defeito ósseo, de maneira a evitar a exposição da dura-máter; D. Periósteo repousando sobre compressa.

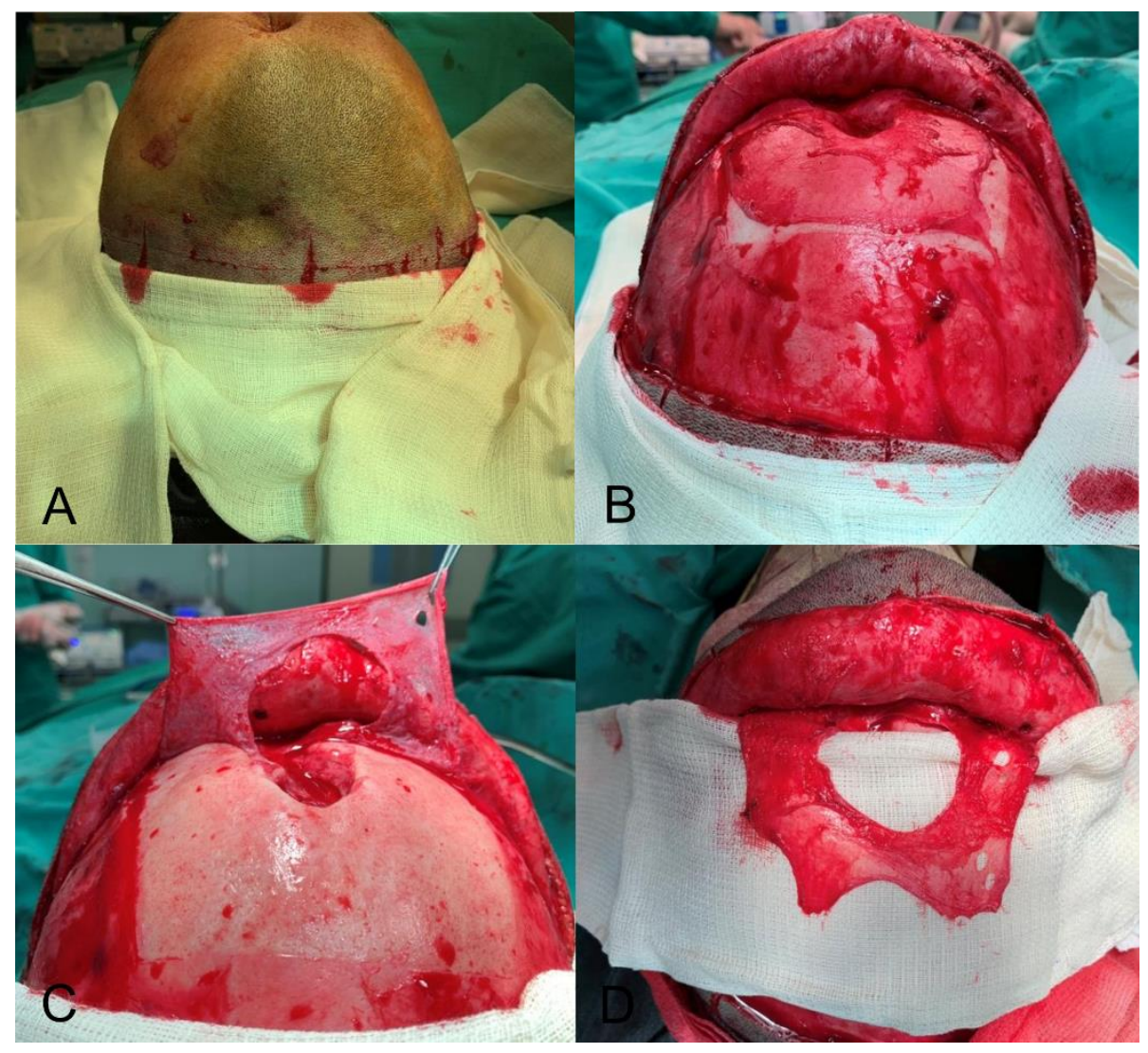

Figura 3 - Acesso bicoronal em sequência: A. Marcação da incisão bicoronal posterior ao "pico de viúva", com marcações verticais para posterior reposicionamento do retalho; B. retalho bicoronal evertido, de maneira que o periósteo foi mantido em posição, e incisado apenas na área de interesse cirúrgico; C. Elevação do periósteo, havendo manutenção do contorno do defeito ósseo, de maneira a proteger e evitar possível exposição da dura-máter; D. Periósteo em evidência, mantendo o contorno da área de defeito ósseo.

Então a tela de titânio de tamanho equivalente ao defeito foi selecionada e modelada, deixando suas arestas circulares, de maneira a evitar exposição transcutânea, e sendo fixada em região de osso saudável, com parafusos de $4 \mathrm{~mm}$ do sistema 1.5 . Na Figura 4. A. Tela de titânio sendo cortada no formato adequado, sobre o template utilizado para a medição; B. Excesso removido e embalagem estéril de fio de sutura utilizada como template. Após a fixação a placa, o retalho coronal foi reposicionado, de maneira a checar o contorno anatômico da fronte do paciente, o contorno foi considerado aceitável, e assim a malha foi recoberta com o periósteo da região adjacente. 0 retalho foi levado de volta à sua posição inicial, suturado em planos, não foi instalado dreno, evidente nas Figuras C e D. 


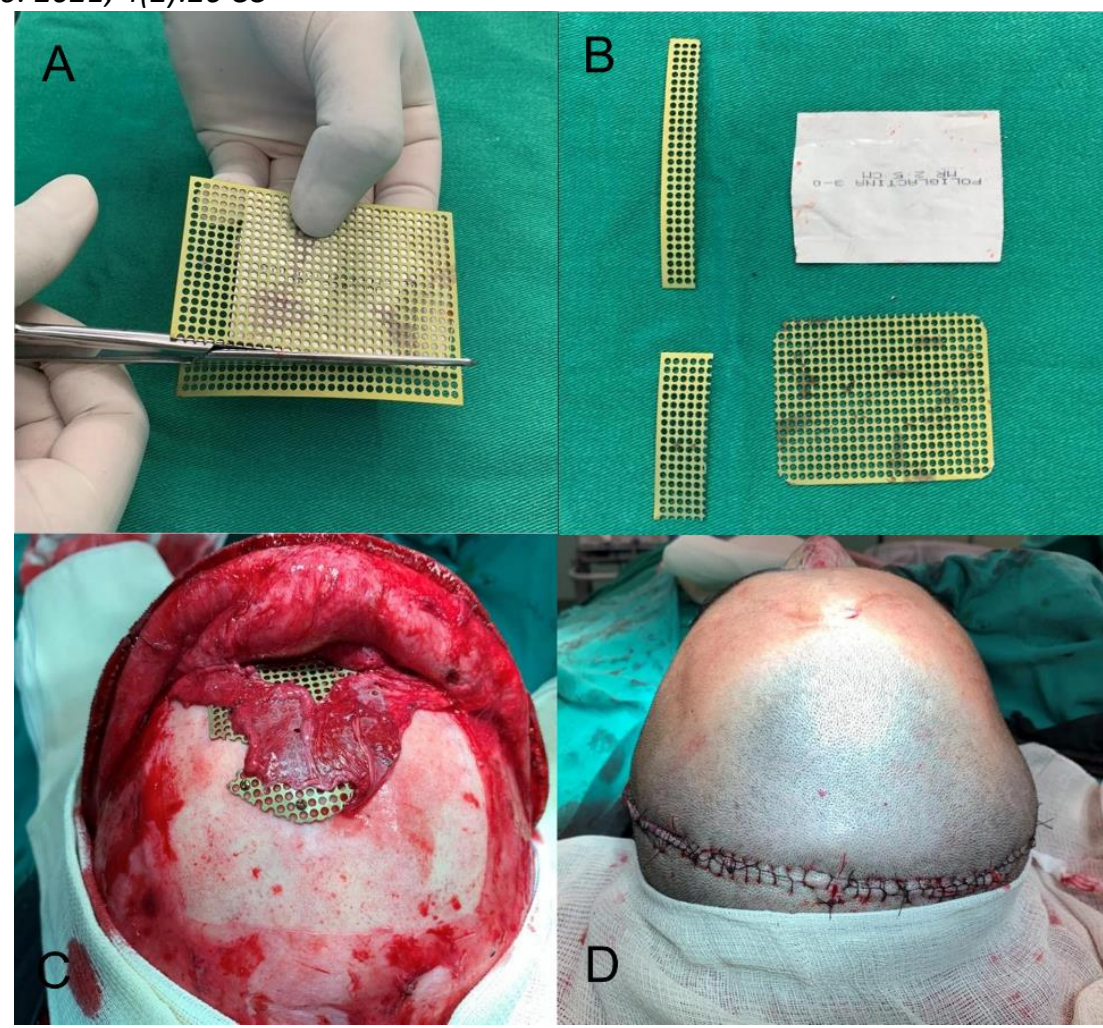

Figura 4 - A. Utilização de um template para o recorte da tela no formato apropriado; B. O template utilizado pode ser a embalagem estéril do fio de sutura a ser utilizado; C. Tela de titânio já recortada e fixada em posição, sendo recoberta pelo periósteo; D. Suturas em posição.

Nas Figuras 5. A e B é possível observar a melhora do aspecto clínico do paciente, que ainda se encontrava em 3 o dia de pós-operatório, tendo uma melhora precoce do quadro cirúrgico. Nas Figuras 6 . A, B e C observamos a presença da tela de titânio de reconstrução em posição.

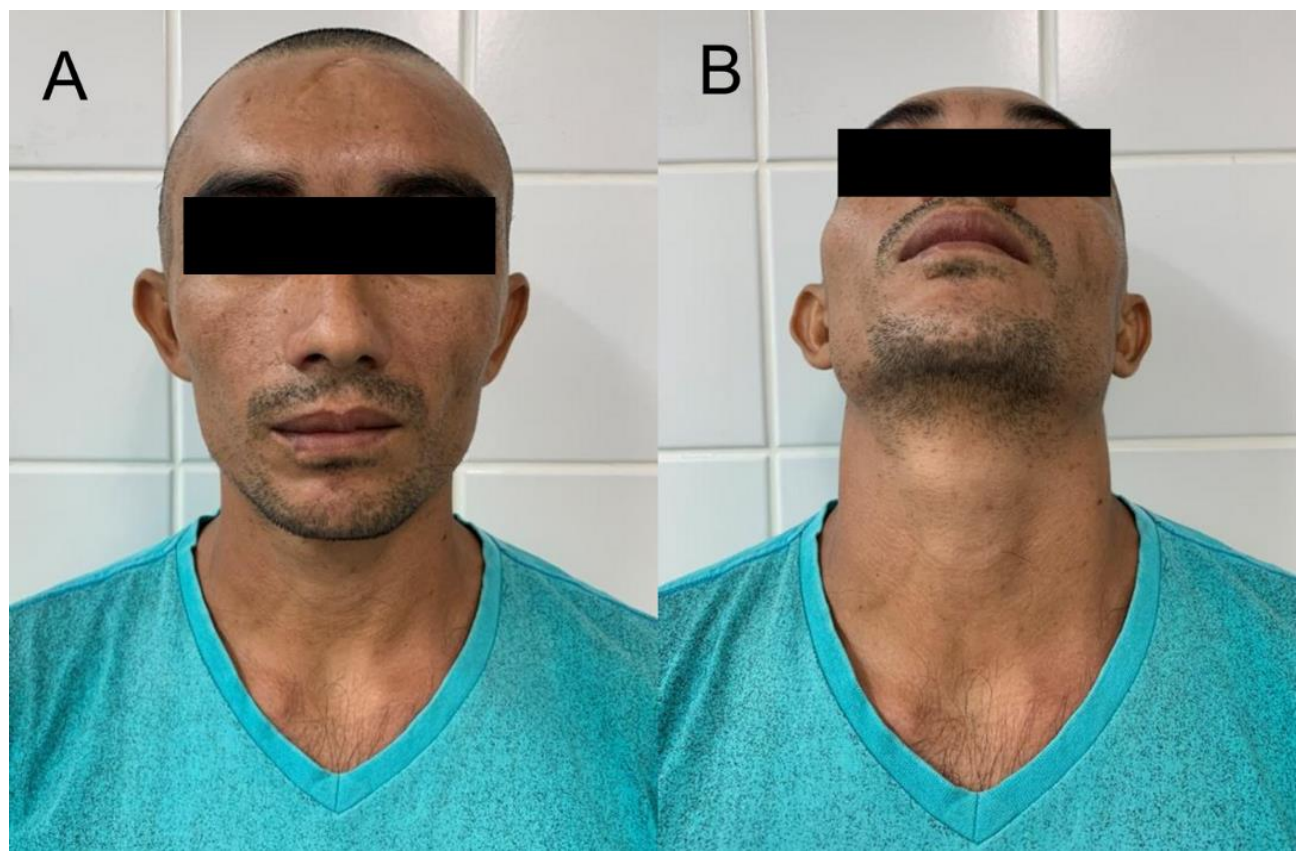

Figura 5 - Paciente em 3o dia de pós-operatório, evidenciando contorno satisfatório da região frontal. 


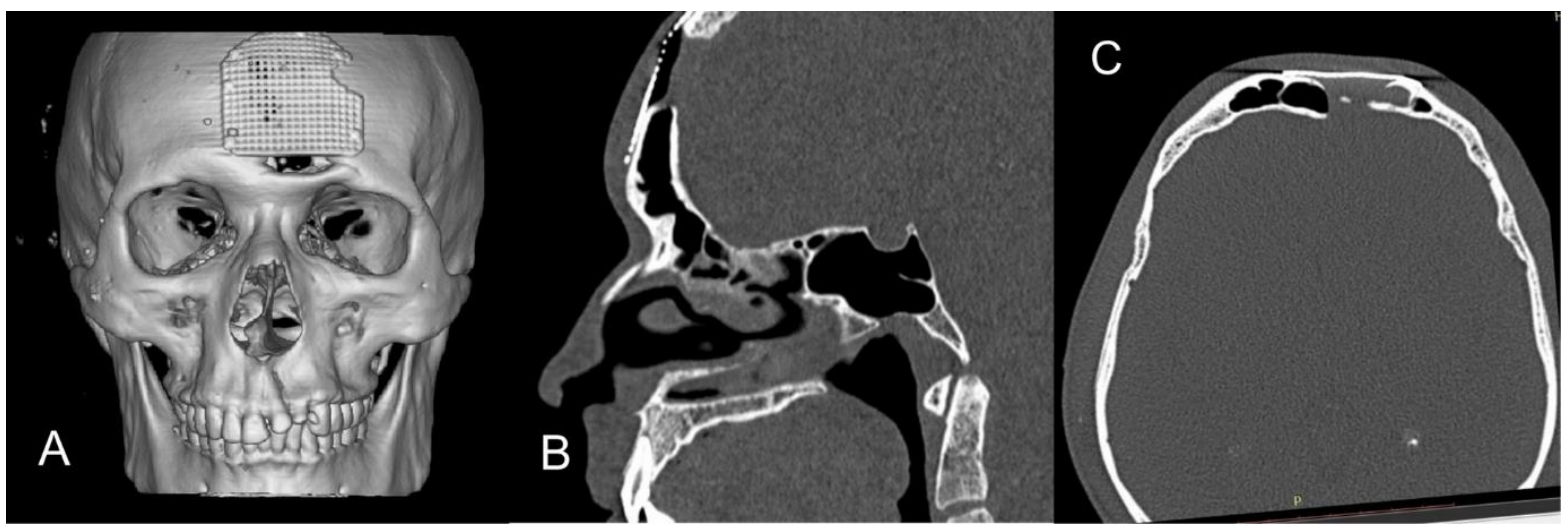

Figura 6 - Imagens de tomografia computadorizada, pós-operatória, evidenciando o posicionamento da tela de titânio da reconstrução realizada. A. Imagem de reconstrução 3D; B. Corte sagital e C. Corte axial.

\section{RESULTADOS E DISCUSSÃO}

O período ideal que se deve aguardar para realização da cranioplastia após uma craniectomia descompressiva é objeto de muitos estudos. Bjornson e colaboradores8 em estudo publicado em 2019, consideram uma reconstrução precoce aquela realizada de 6 semanas a 3 meses após a cirurgia descompressiva, da maneira que 3 meses é a divisão entre precoce e tardia. Noventa paciente foram objeto desse estudo, 89 receberam tela de titânio customizada e um paciente recebeu uma prótese de PEEK (poli-éter-cetona); destes, 66 pacientes (73\%) foi submetido à cranioplastia tardia (mais de 3 meses), e 24 (27\%), cranioplastia precoce. A taxa de complicações foi de $21 \%$ no grupo precoce, e $30 \%$ no grupo tardio, e as principais complicações foram infecção (8\% precoce e 13\% tardio, hidrocefalia, pneumoencéfalo sintomático, hematoma pós-operatório, e incompatibilidade estética. Os autores defendem que a cranioplastia precoce tem os melhores resultados.

No entanto, Madare e colaboradores ${ }^{(1)}$ em estudo de 2019, performaram cranioplastia com malha de titânio em um grupo de 100 pacientes, todos considerados tardios ( 1 a 6 anos após a cirurgia descompressiva), apresentando uma taxa de complicações de $5 \%$ (05 pacientes), havendo três casos de exposição da tela, um seroma tardio e um caso onde houve necessidade de reposicionamento da placa. Os autores defendem que os principais critérios para realização da cranioplastia sejam ausência de feridas abertas ou contaminadas no crânio, ausência de convulsões nos últimos 06 meses, e o paciente não apresentar comorbidades significativas.

O caso apresentado foi executado cerca de 13 meses após a ocorrência do trauma ao paciente, levando em consideração a ausência de sintomas neurológicos, tais como crises convulsivas, ausência de feridas ou sinais clínicos de infecção na região do defeito em fronte, e estado clínico geral do paciente favorável a realização de um procedimento cirúrgico eletivo e de magnitude.

Cho e Kang ${ }^{(5)}$ listam as principais razões pelas quais a cranioplastia deve ser executada, após uma creniectomia: proteção do encéfalo, visto que a ausência de algum segmento ósseo da abóboda craniana deixa o cérebro suscetível à injúrias externas; restabelecer uma aparência natural ao contorno do crânio; prevenção da síndrome da trefinado, a qual é caracterizada pela deterioração neurológica após a remoção de uma parte do osso do crânio.9 Além dessas, são ressaltadas melhoras no metabolismo cerebral da glicose, na capacidade de reserva vascular cerebral, na regulação do fluxo sanguíneo postural e na circulação do fluido cerebroespinhal.

Zanott e colaboradores ${ }^{(10)}$ realizaram ampla revisão sistemática de literatura quanto aos materiais utilizados no procedimento de cranioplastia: o osso autógeno é considerado padrão outro, apesar da morbidade ao sítio doador, e da taxa de reabsorção de 3 a $22 \%$ encontrada, sendo mais comum de ocorrer 
quando sua área ultrapassa $75 \mathrm{~cm}^{2}$; não houve consenso entre os resultados apresentados sobre a Hidroxiapatita, sendo descrita como passível de osteointegração, apesar da discordância de Frassanito e colaboradores; Poliétercetona ou PEEK é descrito como material de força e rigidez similares ao osso cortical, sendo indicada em defeitos os quais seriam muito extensos para a utilização de osso autógeno; descrito como uma resina acrílica moldável, a qual apresenta força similar a do osso, o Polimetilmetacrilato ou PMMA não é indicado para ser utilizado como substituto ósseo, pois interfere na osteocondução e vascularização, não interage com os tecidos circundantes, e estar suscetível à índices mais altos de infecção; o Titânio é considerado uma excelente opção em termos de força, baixos índices de infecção, alta biocompatibilidade, biologicamente inerte, além de outras características favoráveis, sua principal desvantagem seria a condutibilidade térmica. De qualquer maneira, os autores concluem ressaltando como principal norteador para a escolha do material sendo a decisão do cirurgião.

O paciente em questão, objeto deste relato de experiência, está em acompanhamento ambulatorial há 1 ano e 9 meses, não havendo queixas álgicas, estéticas e funcionais no momento, reconstrução utilizando tela de titânio segue curso seguro e previsível, de acordo com a literatura.

\section{CONSIDERAÇÕES FINAIS}

As cirurgias reconstrutivas dos ossos do crânio permanecem sendo desafiadoras ao cirurgião, pois além da grande necessidade estética, a técnica operatória deve ser cuidadosa e precisa, devido a proximidade com estruturas nobres. A decisão da oportunidade cirúrgica deve levar em consideração fatores como o estado neurológico do paciente, o período da última abordagem e as suas condições sistêmicas, não sendo indicadas intervenções em pacientes com quadros convulsivos e alterações metabólicas. A escolha do material deve ser guiada pela experiência do cirurgião, risco de infecção, e disponibilidade do mesmo. O titânio mostra-se como material biocompativel, com baixas taxas de infecção e outras comorbidades. A cranioplastia para reconstrução do osso frontal possibilita o restabelecimento estético e funcional nos casos de defeitos extensos onde a perda do arcabouço de proteção do encéfalo além de alteração do contorno do terço superior da face gera também uma susceptibilidade de injúrias as estruturas intracranianas. Para o caso abordado aqui a técnica cirúrgica e o material utilizado na reconstrução favoreceram a harmonia facial e ganho funcional, suprindo a demanda relatada pelo paciente, bem como, não apresentou quaisquer complicações durante o pósoperatório imediato e tardio.

\section{REFERÊNCIAS}

1. Madaree A, Moyeni N, Le Roux PAJ, Pillay T. Use of Stock Titanium Mesh Plates in Cranioplasty. J Craniofac Surg. 2019 Nov-Dec;30(8):2341-4. Doi: $10.1097 /$ SCS.0000000000005778.

2. Chattopadhyay C. Reconstruction of Acquired Frontal Bone Defects Using Titanium Mesh Implants: A Retrospective Study. J Maxillofac Oral Surg. 2019 Mar;18(1):34-9. Doi: 10.1007/s12663-018-1083-6. Epub 2018 Jan 22. PMID: 30728689; PMCID: PMC6328812.;

3. Sakat MS, Kilic K, Altas E, Gozeler MS, Ucuncu H. Comminuted Frontal Sinus Fracture Reconstructed With Titanium Mesh. J Craniofac Surg. 2016 Mar;27(2):e207-8. Doi: $10.1097 /$ SCS.0000000000002466.

4. Piazza M, Grady MS. Cranioplasty. Neurosurg Clin N Am. 2017 Apr;28(2):257-65. Doi: 10.1016/j.nec.2016.11.008. PMID: 28325460.

5. Cho YJ, Kang SH. Review of Cranioplasty after Decompressive Craniectomy. Korean J Neurotrauma. 2017 Apr;13(1):9-14. doi: 10.13004/kjnt.2017.13.1.9. Epub 2017 Apr 30. PMID: 28512612; PMCID: PMC5432454.

6. Brown DA, Wijdicks EF. Decompressive craniectomy in acute brain injury. Handb Clin Neurol. 2017;140:299- 
318. doi: 10.1016/B978-0-444-63600-3.00016-7. PMID: 28187804.

7. Hitoshi Y, Yamashiro S, Yoshida A, Mukasa A. Cranial Reconstruction with Titanium Mesh for Open Depressed Skull Fracture in Children: Reports of Two Cases with Long-term Observation. Kurume Med J. 2020 Jul 1;66(1):77-80. Doi: 10.2739/kurumemedj.MS661011. Epub 2020 May 1. PMID: 32378531.

8. Delaney SW. Treatment strategies for frontal sinus anterior table fractures and contour deformities. J Plast Reconstr Aesthet Surg. 2016 Aug;69(8):1037-45. doi: 10.1016/j.bjps.2016.06.006. Epub 2016 Jun 18. PMID: 27345471.

9. Ellis II E, Zide MF. Surgical approaches to the facial skeleton. Wavery Co., 2 ed., 2006.

10. Bjornson A, Tajsic T, Kolias AG, Wells A, Naushahi MJ, Anwar F, Helmy A, Timofeev I, Hutchinson PJ. A case series of early and late cranioplasty-comparison of surgical outcomes. Acta Neurochir (Wien). 2019
Mar;161(3):467-72. doi: 10.1007/s00701-019-03820-9. Epub 2019 Feb 4. PMID: 30715606; PMCID: PMC6407742.

11. Santos A et al. Síndrome do trefinado: relato de caso. Arq Bras Neurocir. 2015; 34:327-30.

12. Zanotti B, Zingaretti N, Verlicchi A, Robiony M, Alfieri A, Parodi PC. Cranioplasty: Review of Materials. J Craniofac Surg. 2016 Nov;27(8):2061-72. doi:

10.1097/SCS.0000000000003025. PMID: 28005754.

Fontes de financiamento: Não

Conflito de interesse: Não

Recebido: $12 / 05 / 2021$

Aprovado: 08/06/2021

Publicação: 29/10/2021

Endereço para correspondência: Laís Inês Silva Cardoso. Residente em Cirurgia e Traumatologia Bucomaxilofacial (HU-UFPI) Piauí, Brasil. E-mail: laisinescardoso@gmail.com 